\title{
Evaluation of the Adhesion and Performance of Natural Consolidants for Cotton Canvas Conservation
}

\author{
Alexandra Bridarolli, ${ }^{* \dagger \odot}$ Marianne Odlyha, ${ }^{\dagger}$ Oleksandr Nechyporchuk, ${ }^{\S} \# \oplus$ Krister Holmberg, ${ }^{\S}$ \\ Cristina Ruiz-Recasens, "Romain Bordes, ${ }^{\S(0)}$ and Laurent Bozec ${ }^{\dagger, \perp}$ \\ ${ }^{\dagger}$ UCL Eastman Dental Institute, 256 Grays Inn Road, London WC1X 8LD, U.K. \\ ${ }^{\ddagger}$ Department of Biological Sciences, Birkbeck College, Malet Street, Bloomsbury, London WC1E 7HX, U.K. \\ ${ }^{\S}$ Department of Chemistry and Chemical Engineering, Chalmers University of Technology, 412 96, Gothenburg, Sweden \\ "Arts and Conservation Department, Fine Arts Faculty, University of Barcelona, C/Pau Gargallo, 4, 08028 Barcelona, Spain \\ ${ }^{\perp}$ Faculty of Dentistry, University of Toronto, 124 Edward Street, Toronto, ON M5G 1X3, Canada
}

Supporting Information

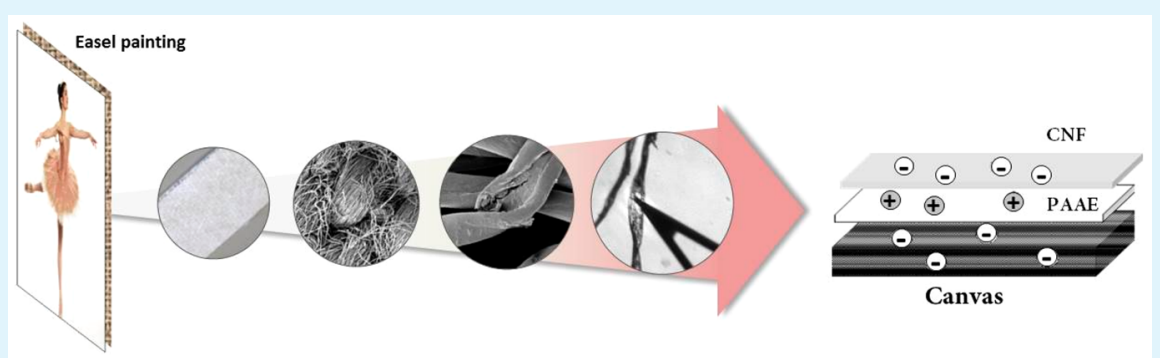

ABSTRACT: Recent developments in paper and canvas conservation have seen the introduction of nanocellulose (NC) as a compatible treatment for the consolidation of historical cellulosic artifacts and manuscripts. However, as part of the assessment of these new materials for canvas consolidation, the adhesion of the consolidation treatment (which takes place between the applied material and the substrate) has not yet been evaluated, and as a result, it is poorly understood by both the scientific and conservation communities. After evaluating the potential of $\mathrm{NC}$ treatments for the consolidation of cotton painting canvas, we investigate a route to promote the interaction between the existing canvas and the nanocellulose treatment, which is in our case made of cellulose nanofibrils (CNF). This was carried out by introducing a cationic polymer, polyamidoamine-epichlorohydrin (PAAE), as an intermediate layer between the canvas and the CNF. The morphological, chemical, and mechanical evaluation of the canvas samples at different relative humidity $(\mathrm{RH})$ levels demonstrated how the adhesion of the added PAAE layer is a dominant factor in the consolidation process. Improvement in the coating of canvas single fibers by the CNF, higher adhesion energy between the canvas fibers and the CNF treatment, and finally overall stronger canvas reinforcement were observed following the introduction of PAAE. However, an increase in mechanical response to moisture sorption and desorption was also observed for the PAAE-treated canvases. Overall, this study shows the complexity of such systems and, as such, the relevance of using a multiscale approach for their assessment.

KEYWORDS: adhesion, nanocellulose, polyamidoamine-epichlorohydrin (PAAE), painting canvas, AFM, DMA-RH

\section{INTRODUCTION}

Like most works of art, easel paintings (i.e., canvas-supported paintings) are prone to damage as they age. One of the most common features of this damage is the loss of strength and elasticity of the canvas, which can then no longer fulfill its supporting role for the paint layer. These changes in mechanical properties are a direct result of both hydrolysis and oxidation of the cellulose of the canvas fibers. In some cases, rupture of the canvas fibers can occur and compromise the structural integrity and therefore the supporting role of the canvas. ${ }^{1}$ Traditionally, the lining of damaged canvas (consisting of "glueing" a new textile layer onto the original canvas) was carried out as a routine procedure ${ }^{1-3}$ as it proved to be an efficient way of strengthening the overall painting. ${ }^{4}$ However, following the 1974 Greenwich conference, the lining technique has been questioned due to the highly invasive nature of the treatment, and the poor physicochemical stability of the adhesives used. $^{5-7}$ Consequently, lining is now only performed as a last resort solution when the canvas has become very brittle or when there are too many tears to use the thread-tothread tear mending approach. ${ }^{8}$

Nanocellulose (NC) is an emergent class of renewable materials comprising cellulosic fibrils/crystals with a diameter in the nanometer range. Using nanocellulose as a consolidant

Received: June 27, 2018

Accepted: August 27, 2018

Published: August 27, 2018 
could be a way to reinforce weak canvases by providing a solution more compatible with the painting's cellulose-based support and could also possibly help avoid a lining. Such an approach would be very welcome by the conservation community. ${ }^{9}$ For the last 15 years, NC has been widely studied as a material for reinforcing paper owing to its mechanical, ${ }^{10,11}$ optical, ${ }^{12}$ and barrier properties, ${ }^{13}$ as well as to its tunability through functionalization. ${ }^{14,15}$ It is expected that its use will grow significantly due to an increased demand for sustainability. In conservation, the use of three types of NC, i.e. the highly crystalline and well-defined rod-like cellulose nanocrystals $(\mathrm{CNC})$, the longer and more flexible cellulose nanofibrils (CNF), and intertwined, long and flexible bacterial nanocellulose (BC), have been investigated as potential consolidants for historical papers, ${ }^{16-18}$ as a filler for common conservation consolidants. ${ }^{19}$ Recently, we showed that CNF and CNC could also be used for consolidation of painting canvases. ${ }^{20,21}$ However, both the limited penetration of the nanocellulose treatment within the canvas mesh and the need for multiple applications to record an improvement in mechanical properties still need to be addressed. The interactions between the existing cellulosic canvas and the nanocellulose-based treatment do require a better understanding to ensure acceptance of the treatments by conservators. Of particular interest is to understand the coupling (morphologically, chemically, and mechanically) of the treatment to the existing cellulosic canvas while being able to respond to environmental variations similarly to the canvas being consolidated.

A good understanding of the response of these various components to moisture uptake is also essential for the validation of this approach in conservation. Several studies have demonstrated the significant role that fluctuations in relative humidity have on the stresses that develop in the layers that make up paintings ${ }^{22-24}$ and in paintings that have been lined. $^{25}$

To address this, here the CNF treatment was applied at a low weight added to the canvas and combined with a cationic polymer, polyamidoamine-epichlorohydrin (PAAE). This strategy is directly inspired by wet-end paper chemistry in which reactive water-soluble polymers are now used routinely to improve the mechanical properties of paper under wet conditions. ${ }^{26}$ The cationic polymer PAAE is one of these commonly used polymers. It bears a protonated quaternary ammonium group of the azetidinium structure that enables strong absorption on cellulose and an azetidinium group which covalently binds to the carboxyl groups present on the pulp fibers forming ester linkages. ${ }^{27-29}$ The resulting tensile strength of PAAE-treated paper rewetted in water was considerably increased. $^{28,29}$ This increase was attributed to intra- and interfiber cross-linking as well as to an increase in cellulose interfiber adhesion. ${ }^{30}$ These mechanisms have been thoroughly described for paper fibers, and here we hypothesize that they would also apply to woven cotton canvases.

The purpose of this study is, therefore, to investigate if PAAE can be used together with CNF to provide an enhanced consolidation while ensuring that the canvas is still able to adapt to changes in its environment (especially relative humidity, RH). We are particularly interested in understanding whether PAAE can promote the adhesion between the treatment and the treated substrate, thus increasing the reinforcing effect of nanocellulose.
In this study, the distribution of the layered treatment onto the canvas surface was examined by scanning electron microscopy (SEM). The adhesion of the treatment (PAAE and $\mathrm{CNF}$ ) to the canvas fibers was characterized by atomic force microscopy (AFM). Complementary to the AFM measurements, information on the surface chemistry of the tested canvases was obtained by X-ray photoelectron spectroscopy (XPS). The direct mechanical consolidation provided by the treatment was measured by tensile testing $(20 \% \mathrm{RH}, 25$ $\left.{ }^{\circ} \mathrm{C}\right)$. Finally, the effect of absorbed water on the mechanical behavior of untreated and treated samples was investigated using dynamic mechanical analysis under controlled and cycling relative humidity (DMA-RH).

\section{MATERIAL AND METHODS}

Materials. CNF prepared from softwood pulp (ca. $75 \%$ pine and $25 \%$ spruce, containing $85 \%$ cellulose, $15 \%$ hemicellulose, and traces of lignin, as determined by the supplier) was provided by Stora Enso $\mathrm{AB}$ (Sweden). An anionic charge density of $3.0 \mu \mathrm{eq} / \mathrm{g}$ at $\mathrm{pH} 4$ was measured for the CNF dispersion using a particle charge detector PCD-02 (Mütek Analytic GmbH, Germany), titrated using poly(diallyldimethylammonium chloride).

PAAE (polyamidoamine-epichlorohydrin) is synthesized from the reaction of epichlorohydrin with polyamidoamine resin, which is formed by the reaction of diethylenetriamine and adipic acid. For this study, we used the commercially available PAAE Eka WS 505 from Akzo Nobel Pulp and Performance Chemicals AB (Gothenburg, Sweden) at $6 \% \mathrm{w} / \mathrm{w}$ in water $(\mathrm{pH} 4)$. A more in-depth study of this product is reported elsewhere. ${ }^{3}$

The investigated cotton canvas was a plain woven fabric of $417 \pm 3$ $\mathrm{g} / \mathrm{m}^{2}$ purchased in Barna Art (Barcelona, Spain). It consisted of 9 and 11 threads $/ \mathrm{cm}$ in the warp and weft directions, respectively. Each thread was made of 2 twisted yarns in both directions.

Methods. Canvas Preparation (Washing and Aging). Before the application of the treatment, pieces of canvas $(70 \mathrm{~mm} \times 80 \mathrm{~mm})$ were artificially aged following a protocol described elsewhere ${ }^{32}$ resulting in a model degraded canvas. The canvases were individually soaked under continuous stirring in a solution of hydrogen peroxide at $35 \mathrm{wt}$ $\%(200 \mathrm{~mL})$ and concentrated sulfuric acid at $95.0-98.0 \%(10 \mathrm{~mL})$ for 3 days at $40{ }^{\circ} \mathrm{C}$. These aging conditions were chosen so that the degree of polymerization (DP) of the canvas reaches a value (500) which is comparable to that measured on original canvas-supported paintings which are described as very fragile by conservators. ${ }^{33}$

Another piece of new canvas (ca. $1 \mathrm{~m}^{2}$ ) was washed in a domestic washing machine at $60{ }^{\circ} \mathrm{C}$ for ca. $40 \mathrm{~min}$ without any detergent and then kept in distilled water in a 1-L double-jacket reactor at $85{ }^{\circ} \mathrm{C}$ with mechanical stirring, followed by rinsing in distilled water at ambient temperature and drying.

Application of the Consolidants. For the application of the consolidants, CNF and PAAE were applied in turn to the surface of the degraded canvas pieces $(8 \mathrm{~cm} \times 8 \mathrm{~cm})$ by spraying. A Cotech Airbrush Compressor AS18B (Clas Ohlson AB, Sweden) at a pressure of 3 bar was used. PAAE $(6 \% \mathrm{w} / \mathrm{w})$ was initially sprayed on the degraded cotton canvas $\left(1.3 \mathrm{~g} / \mathrm{m}^{2}\right)$ which was then set to dry for $2 \mathrm{~h}$ at ambient temperature. Following this step, a dispersion of CNF at $1 \% \mathrm{w} / \mathrm{w}$ in water was deposited via 2 spraying passes with a 20 -min interval, corresponding to $5.8 \mathrm{~g} / \mathrm{m}^{2}$ of deposited material.

Field Emission-Scanning Electron Microscopy (FE-SEM). Prior to imaging, samples $(3 \times 5 \mathrm{~mm})$ were mounted on aluminum stubs (Agar Scientific, Essex, UK) using double-sided carbon tape and sputtered with Gold/Palladium using a Polaron E5000 sputter coater. Following this, surface imaging of the canvas was carried out using a Philips XL30 field emission SEM (FEI, Eindhoven, Netherlands). Regions were selected randomly for imaging, and the data set included representative images obtained at magnifications up to $8000 \times$.

Tensile Testing. For the tensile tests, rectangular samples were cut from different regions of the treated and untreated degraded canvas 


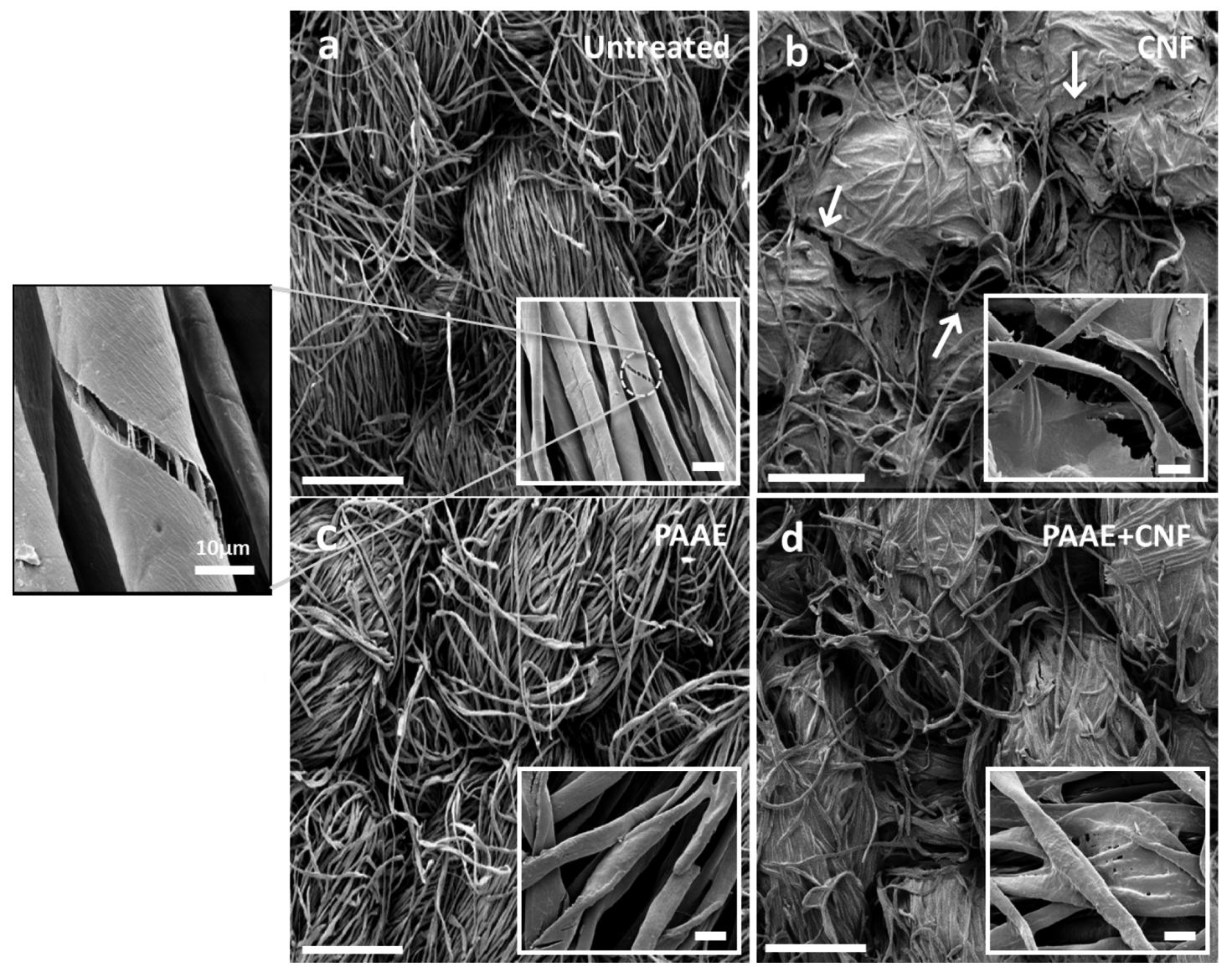

Figure 1. SEM images showing the deposition of the treatment onto the surface (scale bar of $500 \mu \mathrm{m}$ ) and onto the fibers (with scale bar of 20 $\mu \mathrm{m}$ ) for the following: (a) untreated canvas, (b) CNF-treated canvas, (c) PAAE-treated canvas, and (d) PAAE/CNF-treated canvas. Detail of an untreated fiber in (a) shows rupture of the fiber resulting from aging.

pieces parallel to the warp direction. They were cut so that 10 threads were collected in the warp direction and were typically 0.7 (thickness) $\times 7$ (width) $\times 15$ (length) $\mathrm{mm}$ in dimension. The samples were preconditioned at $20 \% \mathrm{RH}$ for at least $24 \mathrm{~h}$ prior to the measurements. The DMA analyzer (Tritec 2000B, Lacerta Technology, UK) was set up for tensile testing. The tests were performed using a free length of $5 \mathrm{~mm}$ in a controlled environment $(20 \% \mathrm{RH}, 25$ ${ }^{\circ} \mathrm{C}$ ). The load was applied at the rate of $0.4 \mathrm{~N} / \mathrm{min}$. This method of measurement enables the evaluation of the mechanical response of the samples at low extensions which are mechanically relevant for paintings. ${ }^{34}$

Dynamic Mechanical Analysis under Controlled Relative Humidity $(D M A-R H)$. To perform the dynamic mechanical analysis, a series of samples were cut from different regions of the treated and untreated degraded canvas pieces parallel to the weft direction. Rectangular samples pieces were cut in order to get 4 threads in the weft direction and were typically 0.7 (thickness) $\times 4$ (width) $\times 15$ (length) $\mathrm{mm}$ in dimension. The samples were preconditioned at $20 \%$ $\mathrm{RH}$ for at least $24 \mathrm{~h}$ prior to measurements. They were then mounted between the clamps of a Tritec 2000B DMA (Lacerta Technology, UK) with a free length of $5 \mathrm{~mm}$ while making sure a pretension load of $1 \mathrm{~N}$ was applied to remove any slack from the samples. The tests were performed at $1 \mathrm{~Hz}$ in the weft direction with a gauge length of 5 $\mathrm{mm}(0.1 \%$ strain). The DMA sample chamber was directly connected to a humidity generator which enabled the controlled application of three successive $\mathrm{RH}$ cycles $20-60-20 \% \mathrm{RH}$ at $25{ }^{\circ} \mathrm{C}$. The rate of change from 20 to $60 \% \mathrm{RH}$ was $4 \% \mathrm{RH} / \mathrm{min}$. The samples were set to equilibrate at 20 or $60 \% \mathrm{RH}$ for $30 \mathrm{~min}$ between each transition.

$X$-ray Photoelectron Spectroscopy (XPS). The XPS surface analysis was carried out for the new, washed and degraded canvases using a Quantum 2000 Scanning ESCA Microprobe (Physical Electronics, ULVAC-PHI, Chanhassen, US). The charge shift was corrected using the binding energy of the aliphatic $\mathrm{C}-\mathrm{C}, \mathrm{C}-\mathrm{H}$ state of $\mathrm{C} 1 \mathrm{~s}$ at $285.0 \mathrm{eV}$ as a reference signal. ${ }^{35}$

Atomic Force Microscopy (AFM). The adhesion forces between the degraded canvas fibers, the nanocellulose fibrils (CNF), and the PAAE were measured using a NanoWizard I AFM system (JPK Instruments, Berlin, Germany) mounted on an Olympus IX71 (Olympus, Tokyo, Japan) inverted microscope. Tipless NPO10 AFM cantilevers with a nominal spring constant of $0.24 \mathrm{~N} / \mathrm{m}$ were used. The probes were functionalized with a silica sphere (probe) of $10-\mu \mathrm{m}$ diameter before being coated with PAAE $(6 \% \mathrm{w} / \mathrm{w})$ or CNF $(1 \% \mathrm{w} / \mathrm{w})$. The freshly coated beads were set to dry for $30 \mathrm{~min}$ prior to the measurement. Initially, the adhesion between the two components PAAE and CNF was tested. Then, tests were conducted using $\mathrm{CNF}$ and PAAE in turn on the degraded canvas fiber. The cotton fiber was fixed on a glass slide at both ends using parafilm in order to avoid contamination expected with the use of glues or solvents. The measurements were performed in contact mode in UHQ water at room temperature. A loading force of $0.7 \mathrm{~N}$ and a constant speed rate of $5 \mu \mathrm{m} / \mathrm{s}$ were used. Force-distance curves were recorded on a minimum of 5 cotton degraded fibers with no less than 7 locations measured for each fiber. For each force-distance curve, the energy of adhesion was measured ${ }^{36}$ by integrating the area under the retraction force-distance curve with the baseline taken at zero force $^{37}$ using the JPK data processing software (JPK Instruments, Berlin, Germany) (Supporting Information, Figure S-1). The adhesion energies calculated were then plotted as a histogram to calculate the median value of energy of adhesion measured.

\section{RESULTS}

Topological Assessment of Canvas Treated with CNF and PAAE/CNF. The SEM images (Figure 1) show that for 
A

(a)

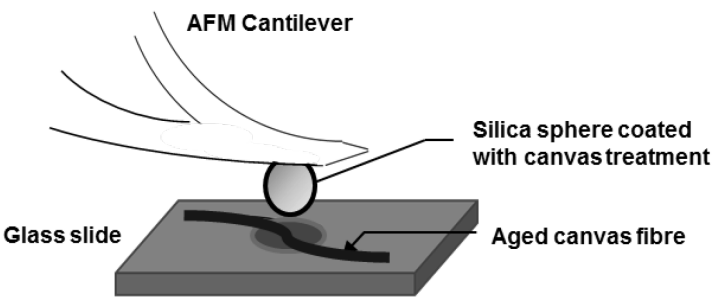

(b)

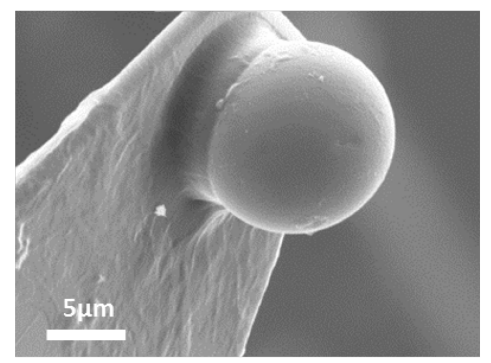

B

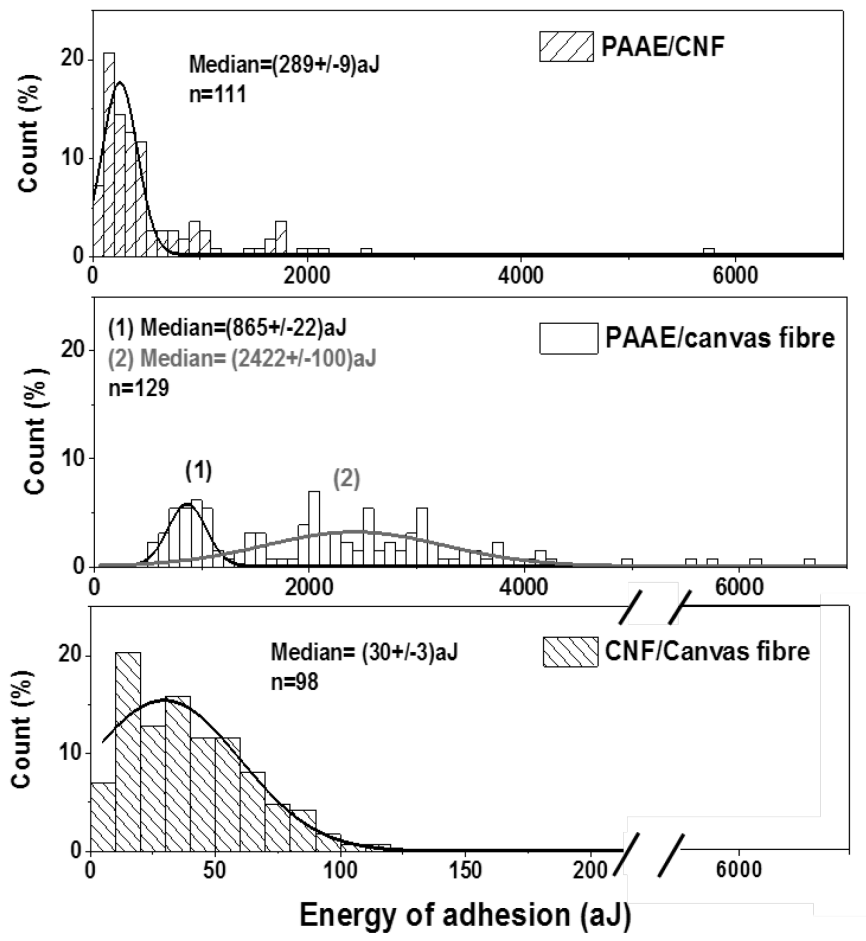

Figure 2. (A) Setup designed for the quantification of the forces developed between the tested treatments and the degraded cotton fiber using AFM (a), SEM image of the bead-functionalized cantilever (b, left), and microscopic image showing the cantilever on a cotton fiber during a measurement (b, right). (B) Distribution of the energy of adhesion calculated between (top) PAAE and CNF, (middle) PAAE and a degraded cotton fiber, and (bottom) CNF and a degraded cotton fiber. Unimodal distributions are seen for the CNF/canvas fiber and the interfaces of the two materials PAAE/CNF, whereas a bimodal distribution was measured for the PAAE/canvas interface. The numbers in parentheses (for PAAE/ canvas fiber) (i.e., (1) and (2)) correspond to the two fitted curves used to determine the energy of adhesion. Median and standard error are given for each Gaussian fitting of the distributions.

the CNF-treated sample, the treatment is visible as a thin coating (around $1-5 \mu \mathrm{m}$ thick) (Figure S2) deposited over the canvas surface threads. The homogeneous coating reduces the overall roughness of the untreated canvas through the formation of a thin film over and between the canvas fibers filling the interfiber and interthread spaces, as previously observed. $^{20,38}$ The treatment is morphologically very compliant, as the CNF tightly wraps around the cotton fibers. Some ruptures of the deposited CNF film can also be seen between the threads for the CNF sample (arrows in Figure 1b). At low magnification, it is possible to observe how the CNF layer used in conjunction with PAAE presents a distribution and surface deposition onto the canvas similar to that of the CNF-only treated sample. However, the surface coverage of the CNF coating of these two samples slightly differs when observed at higher magnification. Several ruptures of the CNF film or its delamination from the cotton fibers can be observed in smaller numbers for the PAAE/CNF treated sample. From the SEM images of the PAAE-only treated sample, the PAAE resin is not seen on the surface of the fibers. As shown previously, ${ }^{39}$ this could result from the absorption of the resin by the canvas fibers. Finally, some interfiber bridges could be observed at high magnification for the PAAE-only treated sample. It is believed that PAAE might promote the formation of interfiber CNF bridges, observed in both cases, and preserve them from rupture.

By applying a higher amount of CNF over the PAAE layer (i.e., 8 applications of CNF corresponding to $26 \mathrm{~g} / \mathrm{m}^{2}$ ), the treatment behaves like a surface coating (Figures S2 and S3).
The improvement provided by the PAAE layer seems to be lost by an excessive accumulation of CNF layers and presents a surface appearance similar to that of the $8 \mathrm{CNF}$-only treated sample.

Adhesion of the Treatment. The improved deposition of nanocellulose onto the canvas promoted by PAAE was further characterized by measuring the adhesion forces developed between the treatments (PAAE and CNF) and the degraded canvas fibers. In painting conservation, macro-peeling tests are most commonly used to assess the adhesive performance of the lining adhesive. For our system, this test presents some limitations such as the small thickness of the PAAE nanocellulose layer $(\sim 5 \mu \mathrm{m})$, which makes peeling from the surface difficult. This problem can be overcome by using an AFM with a functionalized probe (coated with CNF or PAAE) (Figure 2) while at the same time enabling quantification of the adhesion forces at the nanoscale.

The adhesion energy was measured for the different systems in UHQ water in order to replicate the conditions of application of the treatments (all water-based). The results (Figure 2) first show that the adhesion energies measured between PAAE and CNF and between PAAE and canvas fiber are both higher than that measured between CNF and canvas fiber. As such, for the PAAE/CNF and the PAAE/canvas fiber systems, the medians of the distributions (unimodal and bimodal respectively) were calculated to be $E_{\text {adh }}(\mathrm{PAAE} / \mathrm{CNF})$ $=249 \pm 9$ aJ, and $\mathrm{E}_{\text {adh }}^{1}(\mathrm{PAAE} /$ canvas $)=865 \pm 22 \mathrm{aJ}$ and $\mathrm{E}_{\text {adh }}^{2}(\mathrm{PAAE} /$ canvas $)=2422 \pm 100$ aJ, respectively (Table 1$)$. Between CNF and canvas fiber, the adhesion energies remain 
Table 1. Type and Characteristic (Median and Standard Error) of the Distribution of the Energies of Adhesion Measured between the CNF/Canvas Fiber, PAAE/CNF, and PAAE/Canvas Fiber Interfaces

\begin{tabular}{lccc}
\multicolumn{1}{c}{ interface tested } & $\begin{array}{c}\text { PAAE/ } \\
\text { CNF }\end{array}$ & $\begin{array}{c}\text { PAAE/canvas } \\
\text { fiber }^{a}\end{array}$ & $\begin{array}{c}\text { CNF/canvas } \\
\text { fiber }\end{array}$ \\
\hline distribution type & unimodal & bimodal & unimodal \\
energy of adhesion (aJ) & $249 \pm 9$ & (1) $865 \pm 22$ & $30 \pm 3$
\end{tabular}

(2) $2422 \pm 100$

${ }^{a}$ For the PAAE/canvas fiber interface, a bimodal distribution of the energies of adhesion was measured which was deconvoluted into 2 Gaussians defined as (1) and (2).

below 150 aJ with a median at $E_{\text {adh }}(\mathrm{CNF} /$ canvas $)=30 \pm 3$ aJ. The adhesion energies measured when PAAE is introduced as a coupling agent are increased by 1 order of magnitude or more. These results suggest that the cationic sites of PAAE interact with the carboxylate groups present on the degraded cellulose chains of the cotton fibers and the nanocellulose fibrils. ${ }^{40}$ This considerable increase in adhesion confirms that the use of PAAE promotes an enhanced adhesion of CNF onto the canvas, supporting the PAAE/CNF application approach for canvas consolidation.

From these measurements, one can also conclude that CNF does not attach strongly to the canvas fiber. It has been previously stated that the high number of hydroxy groups on the surface of the nanocellulose particles favors the formation of hydrogen bonding with other polymeric materials. ${ }^{41}$ However, since the adhesion measurements need to be performed in a buffer environment (i.e., UHQ water), there is a competition taking place between the hydroxy groups on the CNF surface and water for hydrogen bonding with the hydroxy groups on the cellulose fiber. Hydrogen bonding between fiber surface hydroxy groups and water will take over, which explains the low adhesion energies measured between $\mathrm{CNF}$ and the canvas fibers.

Taking into consideration the shape of the energy distributions (Figure 2), one can observe both unimodal and bimodal behavior for the PAAE/CNF and the PAAE/canvas systems, respectively. For the PAAE/CNF system, more than half the measurements give energies of adhesion below $500 \mathrm{aJ}$ (Table 1). The bimodal distribution for the PAAE/canvas fiber system is also much broader. For this system, the values of adhesion energy span the range 610 to 3600 aJ. The question arises whether this wider distribution could result from differences in the state of the degradation across fibers, and/ or from the chemical inhomogeneity of the fibers surface and inherent twisted morphology of cotton fibers. Up to now, no correlation could be made between the area morphology of the fiber or its state of degradation and the energy of adhesion.

Influence of Canvas Surface Chemistry on the Adhesion of the Treatment. The presence of carboxylic groups on the degraded canvas surface was further investigated by analyzing the surface chemistry of new (i.e., "as received"), washed, and degraded cotton canvases using XPS. The quantitative characterization of the surface chemical composition of the samples was obtained from the relative amount of each type of carbon-other atom interactions $(\mathrm{C}(1 \mathrm{~s}))$ and the $\mathrm{O} / \mathrm{C}$ atomic ratio. The characteristic signature of cellulose consists of the $\mathrm{C}-\mathrm{O}$ and $\mathrm{O}-\mathrm{C}-\mathrm{O}$ bonded carbon atoms, which correspond in XPS spectra to peaks at binding energies of 286.6 and $288 \mathrm{eV}$, respectively, with a relative intensity of
5:1. ${ }^{42,43}$ However, noncellulosic $\mathrm{C}-\mathrm{C}$ carbon is always present due to the presence of impurities (which have been previously mentioned and detected on other cotton fabrics ${ }^{44}$ ) such as fatty acids, alcohols, alkanes, esters, and glycerides.

The results (Table 2) show an increase in the $\mathrm{O} / \mathrm{C}$ ratio between the new and the washed sample from 0.14 to 0.21 as

Table 2. XPS Data of the "As Received" Cotton Canvas before (New) and after Washing Step (Washed) and after Aging Step (Degraded)

\begin{tabular}{|c|c|c|c|c|c|c|}
\hline \multirow[b]{3}{*}{ sample } & \multirow[b]{3}{*}{$0 / \mathrm{C}$} & \multicolumn{5}{|c|}{$\mathrm{C}(\mathrm{ls})$ binding energy $(\mathrm{eV})$} \\
\hline & & 285.0 & 286.0 & 286.6 & 288.1 & 289.0 \\
\hline & & $\begin{array}{c}\mathrm{C}-\mathrm{C} \\
\text { or } \\
\mathrm{C}-\mathrm{H}\end{array}$ & $\begin{array}{c}\mathrm{C}- \\
\operatorname{coo}(-)\end{array}$ & $\begin{array}{c}\mathrm{C}-\mathrm{OH} \\
\text { or } \mathrm{C}- \\
\mathrm{O}-\end{array}$ & $\begin{array}{c}\mathrm{O}-\mathrm{C}- \\
\mathrm{O} \text { or } \\
\mathrm{C}=\mathrm{O}\end{array}$ & $\mathrm{C}=\mathrm{O}-$ \\
\hline new & 0.14 & 74.9 & 0 & 17.89 & 5.1 & 2.2 \\
\hline washed & 0.21 & 67.5 & 0 & 23.2 & 6.4 & 2.9 \\
\hline degraded & 0.30 & 47.1 & 3.8 & 35.9 & 9.5 & 3.8 \\
\hline
\end{tabular}

well as between the washed and the degraded sample from 0.21 to 0.30 . This increase is likely to result from the removal of impurities present on the canvas surface. This removal can also be followed by the reduction in intensity of the carbon peak found at the binding energy $285.0 \mathrm{eV}$ and which corresponds to $\mathrm{C}-\mathrm{C}$ and $\mathrm{C}-\mathrm{H}$ bonds. The area of the peak, assigned by deconvolution to $285.0 \mathrm{eV}$, decreases from $74.9 \%$ calculated for the new canvas to $67.5 \%$ and $47.1 \%$ for the washed and degraded canvas, respectively (Table 2, Figure 3 ). The surface concentration of these impurities could be reduced, but even after bleaching or scouring of the fabric, noncellulosic material residue could still be detected. It is not clear whether the impurities removed from the canvas are inherent to the cotton used or in the manufacturing process (e.g., cellulose wax often used in the weaving process). ${ }^{45}$ It was previously reported that upon drying from water, surface adaptation of cellulose occurs to minimize its surface free energy, resulting in adsorption of a high amount of airborne contaminants on the surface, seen as the $\mathrm{C}-\mathrm{C}$ carbon peak in XPS data. ${ }^{46}$ Because XPS is a surface technique, it is therefore not possible to determine the exact amount of impurity elimination upon washing and accelerated aging. Nevertheless, the canvas samples washed and degraded seem thus to offer a purer cellulosic surface with fewer impurities than the new canvas as indicated by the increase of the spectral intensity at $286.6 \mathrm{eV}$ attributed to $\mathrm{C}-\mathrm{O}$ bonds.

Finally, the degradation procedure used appears also to modify the chemical structure of the cotton canvas cellulose. The formation of carboxylic acid species at the canvas surface, seen at a $\mathrm{C}(1 \mathrm{~s})$ binding energy of $289.0 \mathrm{eV}$, suggests that as oxidation and acid-catalyzed chain scission increase with treatment time, carboxyl groups are formed ${ }^{47}$ on the cellulose backbone. This was expected from the design of the experiment $^{33}$ and was confirmed by XPS analysis.

Contribution of the Treatment toward Increased Stiffening of the Canvas. Regarding mechanical reinforcement, the consolidation provided by the treatment with $\mathrm{CNF}$ was also improved by the use of PAAE. Paintings are usually restretched up to $1-2 \%$ in extension. ${ }^{34}$ In this range, the stiffening of the multilayered PAAE/CNF sample was shown by an increase in Young's modulus (calculated from the slope of the stress-strain curve) from $1.9 \pm 0.7$ to $9.2 \pm 3.3 \mathrm{MPa}$ measured before and after application (Figure 4, Table 3). An 

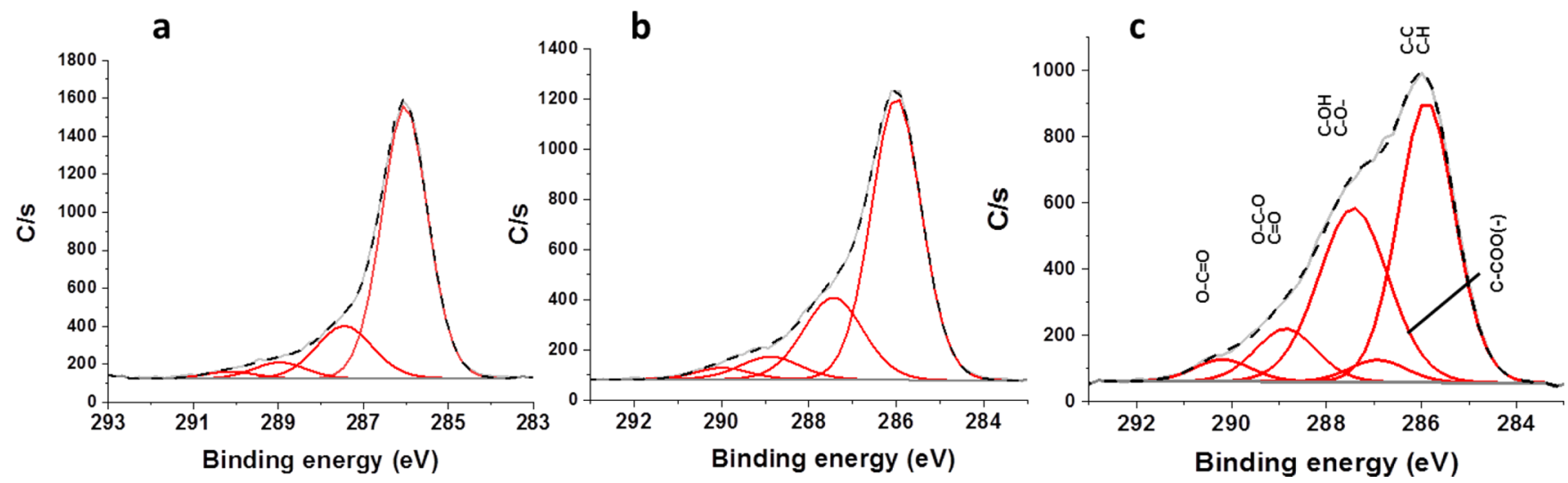

Figure 3. XPS spectra of the new (a), washed (b), and degraded (c) cotton canvases (in gray) with the deconvolution curves (in red) and the resulting fitting curve (in black).

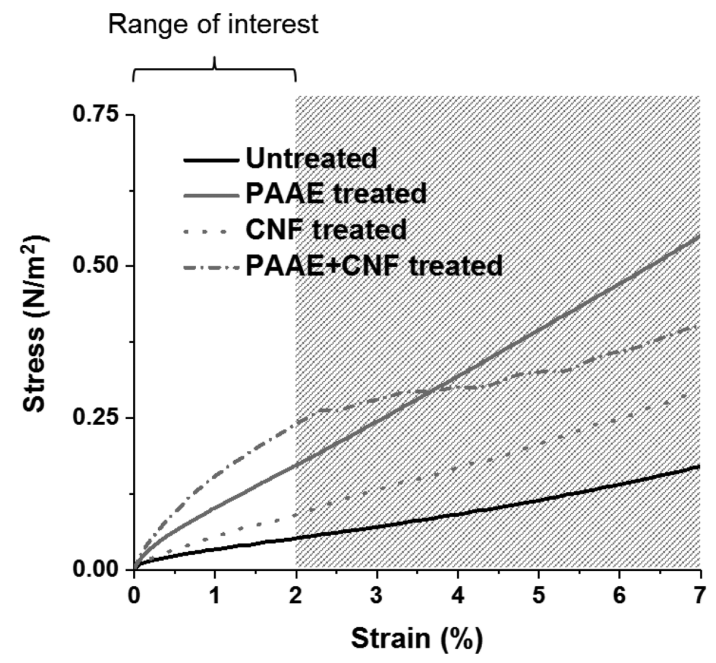

Figure 4. Tensile behavior of untreated and treated samples at $20 \%$ $\mathrm{RH}$ showing the increase in stiffness of the PAAE/CNF sample in the range of interest (i.e., strain at which paintings are usually restretched).

Table 3. Young's Moduli at $20 \% \mathrm{RH}$ (Calculated from the Stress-Strain Curves) for Untreated and Treated Samples

\begin{tabular}{lc}
\multicolumn{1}{c}{ sample } & Young's modulus $(\mathrm{MPa})$ \\
untreated canvas & $1.9 \pm 0.7$ \\
PAAE-treated canvas & $6.8 \pm 0.5$ \\
CNF-treated canvas & $2.8 \pm 0.1$ \\
PAAE/CNF-treated canvas & $9.2 \pm 3.3$ \\
\hline
\end{tabular}

increase in stiffness was also measured for the CNF- and PAAE-treated samples for which Young's moduli of $2.8 \pm 0.1$ and $6.8 \pm 0.5 \mathrm{MPa}$ were measured, respectively. For the PAAE-only treated sample, the strong reinforcement measured could result from the inter cross-linking of cellulose by the formation of resin-fiber chemical bonds, and the intra- or selfcross-linking occurring in PAAE when drying. ${ }^{27,31}$ The fact that the reinforcement is weaker for the CNF-only treatment is due to the weaker hydrogen bonds that have formed upon drying of the CNF treatment. The Young's moduli values measured for these samples are, however, lower than the $9.2 \pm$ 3.3 MPa obtained when the treatments were combined. The greater reinforcement reached with the introduction of PAAE is probably related to its function as a coupling agent between $\mathrm{CNF}$ and the cellulosic fiber.

Nonlinear behavior of the stress-strain curve for the PAAE/ CNF treated sample is, however, seen in Figure 4 above $2 \%$ of extension. After $2.5 \%$ in elongation, the tension measured for this sample is stabilized around $0.29 \mathrm{~N} / \mathrm{m}^{2}$ and becomes lower than the one measured for the PAAE-treated sample after 3.8\% elongation. This behavior has been already observed before for samples treated with a higher amount of CNF only. ${ }^{20}$ The irregular slope can be attributed to localized ruptures of the CNF layer sitting on the top canvas fibrils. SEM images, which were taken of the canvas after tensile testing, support this assumption (see Figure 5). The absence of similar drops in tension for the CNF-only treated sample could result from the high brittleness of the CNF layer and the local failures and delamination from the canvas fibers. This was previously observed for the CNF-treated canvas $^{20}$ (see Figure 1). This suggests that the low amount of CNF deposited for 2 applications does not form a continuous and strong enough layer which would support the tension applied to the canvas during handling. Thus, reinforcement seems only provided to the canvas when the adhesion between the nanocellulose and the canvas is improved (case of PAAE/CNF) or when a higher amount of CNF is applied. ${ }^{20}$ It should, however, be noted that the reinforcement in the case of the PAAE/CNF sample is limited to the low elongation values (Figure 4). These values remain in the range identified by conservators as appropriate for canvas retensioning (i.e., $1-2 \%$ in elongation). ${ }^{34}$ However, if the tension applied during retensioning should exceed $2 \%$ extension, the brittleness of the CNF coating should always be considered as a possible limitation of this treatment.

Response to Moisture. When considering novel treatments, it is essential to ensure that the introduction of these new materials does not interfere with the natural response of the canvas under variable environmental conditions. Figure 6 shows the mechanical response fingerprint of an untreated cotton canvas subjected to relative humidity fluctuations. The DMA-RH curve indicates that the canvas tends to stiffen (higher storage modulus $E^{\prime}$ ) at a lower level of relative humidity and to become more viscoelastic at higher humidity levels (lower $E^{\prime}$ ). This behavior is directly related to the canvas material (i.e., cotton) and to the weaving process. Exploring this in more detail, quantification of the mechanical stress experienced by the samples was performed by calculating the 

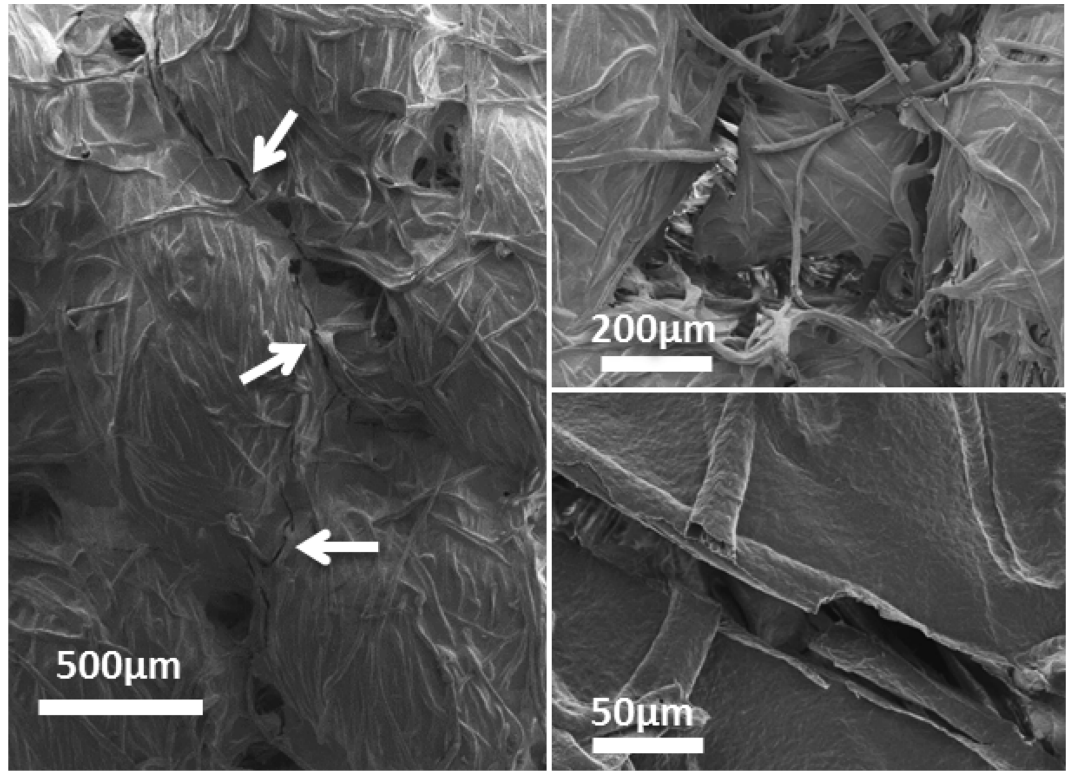

Figure 5. SEM images of a PAAE/CNF-treated canvas after tensile test measurement showing zones of rupture of the superficial CNF film: ruptures in the interthreads spaces and along the canvas fibers (see arrows).

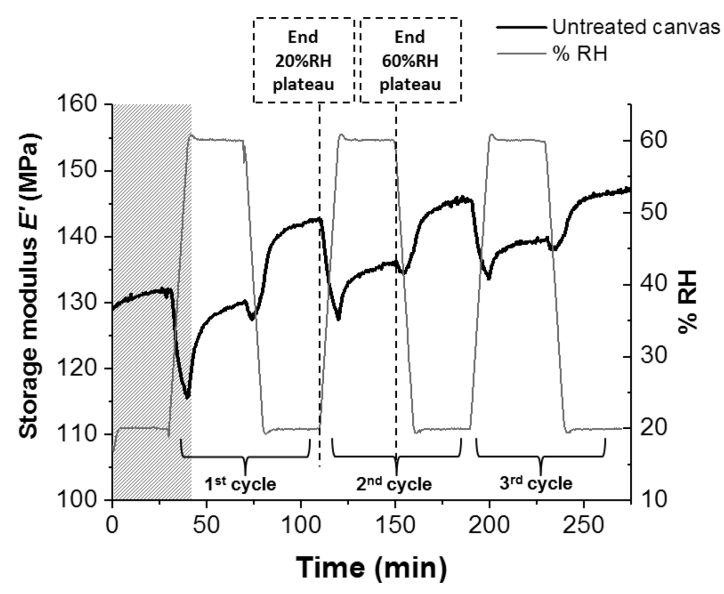

Figure 6. Mechanical response $\left(E^{\prime}\right)$ of an untreated canvas to $\mathrm{RH}$ cycling $(20-60-20 \% \mathrm{RH})$ over time. Variations in \%RH and resulting changes in $E^{\prime}$ are highlighted by the dotted lines placed at the end of the 20 (first RH cycle) and $60 \% \mathrm{RH}$ plateaus (second $\mathrm{RH}$ cycle).

difference between storage moduli $\left(E^{\prime}\right)$ at $60 \% \mathrm{RH}$ and at $20 \%$ $\mathrm{RH}$ (end plateau values as described in Figure 7).

The results are shown for 3 cycles of change in RH. Using this approach, one obtains the response to moisture by the samples after treatment and the impact of the treatment on their resulting mechanical behavior. As shown in Figure 7, for all the samples the difference between $E^{\prime}(60 \% \mathrm{RH})$ and $\mathrm{E}^{\prime}(20 \% \mathrm{RH})$ decreases with time. For sample PAAE/CNF, the difference in $E^{\prime}$ goes from 16.5 to $13.1 \mathrm{MPa}$ from the first to the third cycle. This decrease might relate to the structural stabilization of the fabric upon moisture sorption and desorption. Diffusion of moisture in fabrics is known to follow different paths inducing changes in the size of the cellulose fibers. Although fibers swell during moisture sorption and considering that these fibers are not completely elastic, one can record an incomplete recovery of the fibers' mechanical properties after desorption. A hysteresis between sorption and desorption thus arises. ${ }^{48}$ For our sample, such a hysteresis at a slower rate of moisture desorption was found in the preliminary investigations (Figure S4).

As shown in Figure 7, the use of PAAE tends to increase the canvas response to moisture. For the third cycle, the difference in $E^{\prime}$ calculated for the PAAE- and PAAE/CNF-treated samples reached $10.6 \pm 0.5$ and $13.1 \pm 0.4 \mathrm{MPa}$, respectively. That is almost twice as much as the values obtained (again for the third cycle) for the untreated and the CNF-treated samples, which gave values of $6.9 \pm 1.5$ and $5.1 \pm 1.0 \mathrm{MPa}$, respectively. Upon adding PAAE as an intermediate layer, the mechanical response of the fabric to moisture is amplified, leading to the higher variations in $E^{\prime}$ observed across the $\mathrm{RH}$ cycles. However, it has been shown that the mechanism behind the wet-strength properties of PAAE in paper results from cross-linking of the resin upon drying which then offers a protective network of cross-linked molecules for the fiberfiber contacts. ${ }^{49}$ PAAE has also been shown to efficiently limit the loss in strength of paper under wet conditions by increasing the ratio wet/dry strength from $10 \%$ without PAAE to $33 \%$ for $10 \mathrm{mg} / \mathrm{g}$ PAAE per gram of paper pulp. ${ }^{50}$ Even if these mechanisms apply well to paper, they do not seem to apply here for fabrics. This difference in behavior could presumably result from the method of application used (i.e., spraying) or by the limited amount used. The limited penetration of the PAAE into the fibers is also plausible as it is known that penetration can be limited by the composition and structure (pores) of the fibers. ${ }^{39}$ Higher mechanical stability was instead observed for the CNF-only treated sample. The difference in storage modulus $\left(E^{\prime}\right)$ measured for this sample is similar to that for the untreated sample and confirms already published work where the CNF treatment had been applied at a higher amount and by blade-coating. ${ }^{20}$

\section{CONCLUSIONS}

With these results, we show that the application of PAAE as an intermediate layer promotes adhesion of CNF particles onto the canvas fibers by improving their morphological, chemical, and mechanical coupling with the canvas fibers. The improvement can be attributed to nanomechanical analysis 


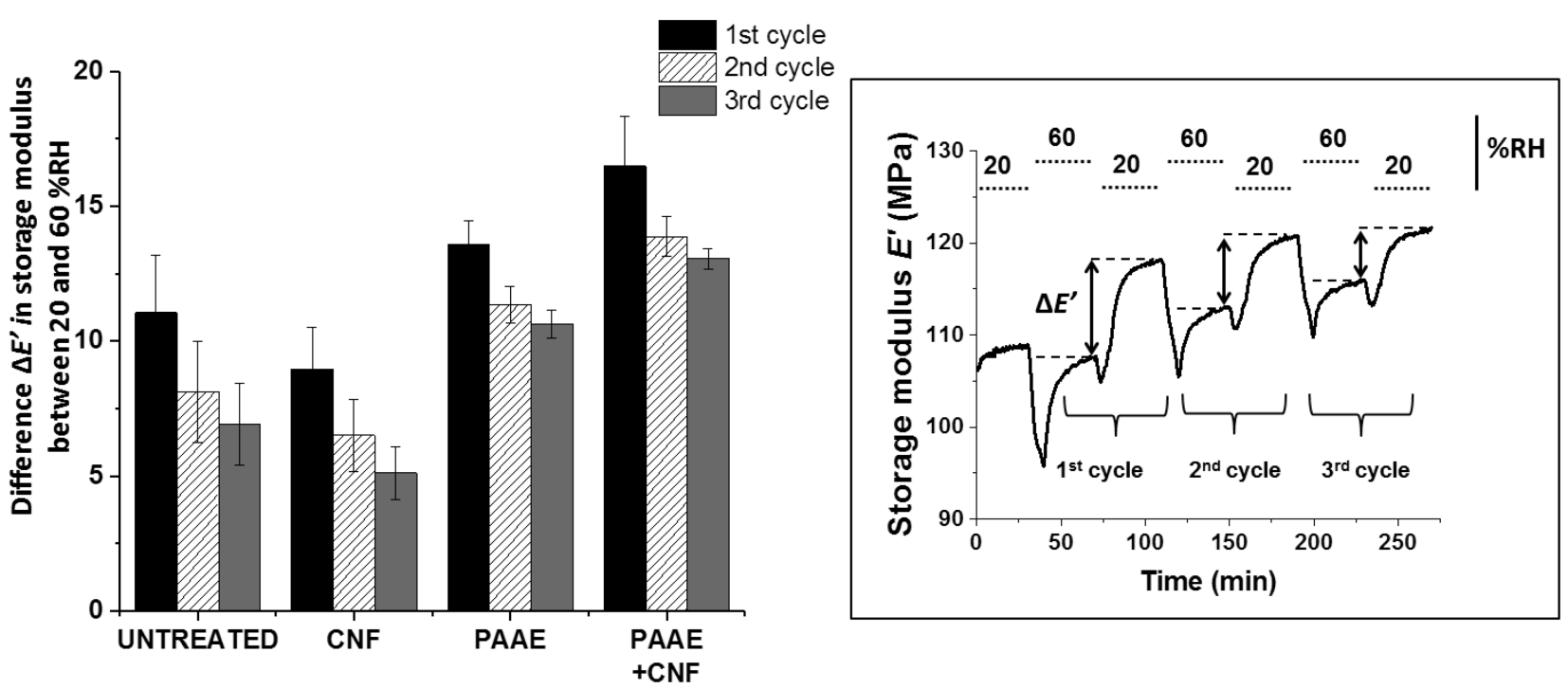

Figure 7. Left: difference $\Delta E^{\prime}$ calculated between storage moduli $\left(E^{\prime}\right)$ measured at the end of the $60 \% \mathrm{RH}$ and $20 \% \mathrm{RH}$ plateaus for each of the 3 $\mathrm{RH}$ cycles. Right: details of a DMA-RH curve showing where the $\Delta E^{\prime}$ values were measured.

of the stronger adhesion taking place both between $\mathrm{CNF}$ and PAAE and between canvas fibers and PAAE. The combination of the strong mechanical properties of CNF and the high adhesion promoting the effect of PAAE makes this multilayered treatment an efficient reinforcement procedure despite the low weight of CNF added. These results confirm the potential of PAAE as an anchor on the canvas for the nanocellulose fibrils. In this work, we have also demonstrated that a severe shortcoming of the use of PAAE for painting conservation is the high mechanical response to moisture to which such treatments give rise. It is, however, yet to be investigated whether the stresses measured will lead to damages to the painting or reduce the stresses already present in the painting materials. ${ }^{22}$

This study also demonstrates the important role of adhesion in the development of new nanocellulose treatments for painting conservation. We foresee that the mechanical performance of nanocellulose as reinforcement material can be greatly improved by combining it with additives that will promote adhesion onto fabrics. Chemical functionalization of nanocellulose might offer an alternative route to the introduction of noncellulosic additives. In the frame of the application of nanocellulose-based treatments in conservation, it is expected that some compromise between good coupling and reversibility will have to be made.

\section{ASSOCIATED CONTENT}

\section{S Supporting Information}

The Supporting Information is available free of charge on the ACS Publications website at DOI: 10.1021/acsami.8b10727.

Figure S1. Principles behind the AFM adhesion measurements and data processing: (a) scheme of the tip movement during a single approach-retract cycle of the AFM $\operatorname{tip}^{37}$ (b), diagram of the resulting force distance curve measured and (c) retract curve showing the area under the curve (shaded area) which was used to calculate the energy of adhesion. Figure S2. SEM images of cross sections of the PAAE/2CNF (a) and PAAE/ 8CNF (b) samples showing thicknesses of the $\mathrm{CNF}$ layer around $1-2$ and $5 \mu \mathrm{m}$, respectively. Figure
S3. Scheme of the multilayered structure of (a) a treated sample with PAAE and CNF and (b) FE-SEM images showing the deposition of the treatment (2 applications (i.e., 2CNF) and 8 applications (i.e., 8CNF) with or without PAAE) onto the surface (scale of $500 \mu \mathrm{m}$ ) and the fibers (scale of $20 \mu \mathrm{m}$ ) of the canvas. Figure S4. Variation in weight measured for an untreated cotton canvas following changes in $\mathrm{RH}$ (ramp and plateaus) (PDF)

\section{AUTHOR INFORMATION}

\section{Corresponding Author}

*alexandra.bridarolli.15@ucl.ac.uk.

ORCID

Alexandra Bridarolli: 0000-0002-0114-751X

Oleksandr Nechyporchuk: 0000-0001-7178-5202

Romain Bordes: 0000-0002-0785-2017

Present Address

\#Swerea IVF, SE-431 22 Mölndal, Sweden.

\section{Author Contributions}

The manuscript was written through contributions of all authors. All authors have given approval to the final version of the manuscript.

\section{Funding}

This study was supported by a cofunding from the H2020 Nanorestart European Project and the Engineering and Physical Sciences Research Council (EPSRC) through the Centre for Doctoral Training In Science and Engineering in Arts, Heritage and Archaeology (SEAHA).

\section{Notes}

The authors declare no competing financial interest.

\section{ACKNOWLEDGMENTS}

We thank Lucia Noor Melita for her support; Adam Strange, Sebastian Aguayo, and Richard Thorogate for the help provided with the AFM measurements; and Asma Buanz for her help with the DVS measurements. Anne Wendel is acknowledged for conducting the XPS analyses and Winnats Scientific Services (UK) for processing the XPS data. 


\section{ABBREVIATIONS}

AFM, atomic force microscopy

$\mathrm{BC}$, bacterial nanocellulose

CNC, cellulose nanocrystals

CNF, cellulose nanofibrils

DMA-RH, dynamic mechanical analysis with controlled relative humidity

FE-SEM, field emission-scanning electron microscopy

NC, nanocellulose

PAAE, Polyamidoamine-epichlorohydrin

$\mathrm{RH}$, relative humidity

XPS, X-ray photoelectron spectroscopy

\section{REFERENCES}

(1) Nicolaus, K. The Restoration of Paintings; Könemann Veragsgesellschaft mbH: Cologne, Germany, 1999. ISBN 3-89508922-2.

(2) Keck, C. Lining Adhesives: Their History, Uses and Abuses. J. Am. Inst. Conserv. 1977, 17 (1), 45-52.

(3) Hackney, S. Paintings on Canvas: Lining and Alternatives. Tate Papers, no. 2. Autumn 2004.

(4) Hackney, S.; Reifsnyder, J.; Marvelde, M.; Scharff, M. In Conservation of Easel Paintings; Hill Stoner, J., Rushfield, R., Eds.; Routledge: London, 2012; pp 412-450.

(5) Duffy, M. A Study of Acrylic Dispersions Used in Treatment of Paintings. J. Am. Inst. Conserv. 1989, 28 (2), 67-77.

(6) Bomford, D.; Staniforth, S. Wax-Resin Lining and Colour Change: An Evaluation. In National Gallery Technical Bulletin, 1981;

Vol 5, pp 58-65. http://www.nationalgallery.org.uk/technicalbulletin/bomforth staniforth1981.

(7) Ploeger, R.; René De La Rie, E.; Mcglinchey, C. W.; Palmer, M.; Maines, C. A.; Chiantore, O. The Long-Term Stability of a Popular Heat-Seal Adhesive for the Conservation of Painted Cultural Objects. Polym. Degrad. Stab. 2014, 107, 307-313.

(8) Ackroyd, P.; Phenix, A.; Villers, C. Structural Treatments for Canvas Paintings in 2002: Summary of Questionnaires Replies. In ICOM Committee for Conservation 13th Triennal Meeting, Rio de Janeiro, 22-27 September 2002: Preprints; Vontobel, R., Ed.; James \& James: London, UK, 2002; pp 321-327.

(9) Oriola, M.; Nualart-Torroja, A.; Ruiz-Recasens, C.; CampoFrancés, G. Canvas Consolidation Survey and Treatment Requirements for the NANORESTART Project. In Proceedings of TechnoHeritage; Cadiz, Eds.; CRC Press/Balkema: Leiden, NL, 2017 (submitted).

(10) Lavoine, N.; Bras, J.; Desloges, I. Mechanical and Barrier Properties of Cardboard and 3D Packaging Coated with Microfibrillated Cellulose. J. Appl. Polym. Sci. 2014, 131 (8), No. 40106.

(11) Dufresne, A. Nanocellulose - From Nature to High Performance Tailored Materials; Walter de Gruyter GmbH \& Co. KG: Berlin, Germany, 2012.

(12) Hu, L.; Zheng, G.; Yao, J.; Liu, N.; Weil, B.; Eskilsson, M.; Karabulut, E.; Ruan, Z.; Fan, S.; Bloking, J. T. Transparent and Conductive Paper from Nanocellulose Fibers. Energy Environ. Sci. 2013, 6, 513-518.

(13) Aulin, C.; Gällstedt, M.; Lindström, T. Oxygen and Oil Barrier Properties of Microfibrillated Cellulose Films and Coatings. Cellulose 2010, 17 (3), 559-574.

(14) Nechyporchuk, O.; Belgacem, M. N.; Bras, J. Production of Cellulose Nanofibrils: A Review of Recent Advances. Ind. Crops Prod. 2016, 93, 2.

(15) Daud, J. B.; Lee, K. In Handbook of Nanocellulose and Cellulose Nanocomposites; Kargarzadeh, H., Ahmad, I., Thomas, S., Dufresne, T., Eds.; Wiley-VCH Verlag: Weinheim, Germany, 2017; pp 101122.

(16) Völkel, L.; Ahn, K.; Hähner, U.; Gindl-Altmutter, W.; Potthast, A. Nano Meets the Sheet: Adhesive-Free Application of Nanocellulosic Suspensions in Paper Conservation. Heritage Sci. 2017, 5, 1.
(17) Dreyfuss-Deseigne, R. Nanocellulose Films in Art Conservation. Journal of Paper Conservation 2017, 18 (1), 18-29.

(18) Santos, S. M.; Carbajo, J. M.; Quintana, E.; Ibarra, D.; Gomez, N.; Ladero, M.; Villar, J. C.; Eugenio, M. E. Characterization of Purified Bacterial Cellulose Focused on its Use on Paper Restoration. Carbohydr. Polym. 2015, 116, 173-181.

(19) Cataldi, A.; Dorigato, A.; Deflorian, F.; Pegoretti, A. Innovative Microcrystalline Cellulose Composites as Lining Adhesives for Canvas. Polym. Eng. Sci. 2015, 55 (6), 1349-1354.

(20) Nechyporchuk, O.; Kolman, K.; Bridarolli, A.; Odlyha, M.; Bozec, L.; Oriola, M.; Campo-Francés, G.; Persson, M.; Holmberg, K.; Bordes, R. On the Potential of Using Nanocellulose for Consolidation of Painting Canvases. Carbohydr. Polym. 2018, 194, 161-169.

(21) Kolman, K. P.; Nechyporchuk, O.; Persson, M.; Holmberg, K.; Bordes, R. Combined Nanocellulose/Nanosilica Approach for MultiScale Consolidation of Painting Canvas. ACS Applied Nano Materials 2018, 1 (5), 2036-2040.

(22) Young, C.; Ackroyd, P.The Mechanical Behaviour and Environmental Response of Paintings to Three Types of Lining Treatment. In National Gallery Technical Bulletin, 2001; Vol 22, p 85. http://www.nationalgallery.org.uk/technical-bulletin/young ackroyd2001.

(23) Mecklenburg, M. F.; Fuster-Lopez, L.; Ottolini, S. In Adhesives and Consolidants in Painting Conservation Barros D'Sa, A., Bone, L., Clarricoates, R., Gent, A., Eds.; Archetype Publications: London, 2012; pp 30-36.

(24) Hendrickx, R.; Desmarais, G.; Weder, M.; Ferreira, E. S. B.; Derome, D. Moisture Uptake and Permeability of Canvas Paintings and their Components. Journal of Cultural Heritage 2016, 19, 445.

(25) Andersen, C. K. Lined Canvas Paintings: Mechanical Properties and Structural Response to Fluctuating Relative Humidity, Exemplified by the Collection of Danish Golden Age Paintings at Statens Museum for Kunst (SMK). Ph.D. Thesis, Royal Danish Academy of Fine Arts, Copenhagen, Denmark, 2013.

(26) Crisp, M. T.; Riehle, R. J. Wet-Strengthening of Paper in Neutral pH Papermaking Conditions. In Applications of Wet-End Paper Chemistry; Thorn, I., Au, C. O., Eka Chemicals Ltd. (Somerset, UK), Eds; Springer Science: Dordrecht, NL, 2009; Ch 7, pp 147169.

(27) Espy, H. H. The Mechanism of Wet-Strength Development in Paper: a Review. Tappi J. 1995, 78 (4), 90-99.

(28) Obokata, T.; Isogai, A. The Mechanism of Wet-Strength Development of Cellulose Sheets Prepared with PolyamideamineEpichlorohydrin (PAE) Resin. Colloids Surf., A 2007, 302 (1), 525531.

(29) Obokata, T.; Yanagisawa, M.; Isogai, A. Characterization of Polyamideamine-Epichlorohydrin (PAE) Resin: Roles of Azetidinium Groups and Molecular Mass of PAE in Wet Strength Development of Paper Prepared with PAE. J. Appl. Polym. Sci. 2005, 97, 2249-2255.

(30) Wang, J.; Yiu, B.; Obermeyer, J.; Filipe, C. D.; Brennan, J. D.; Pelton, R. Effects of Temperature and Relative Humidity on the Stability of Paper-Immobilized Antibodies. Biomacromolecules 2012, $13,559-564$

(31) Siqueira, E. J. Polyamidoamine Epichlorohydrin-based Papers: Mechanisms of Wet Strength Development and Paper Repulping. Ph.D. Thesis, Université Grenoble Alpes, Grenoble, France, 2012.

(32) Nechyporchuk, O.; Kolman, K.; Oriola, M.; Persson, M.; Holmberg, K.; Bordes, R. Accelerated Ageing of Cotton Canvas as a Model for Further Consolidation Practices. J. Cultural Heritage 2017, 28, 183-187.

(33) Oriola, M.; Campo, G.; Ruiz-Recasens, C.; Pedragosa, N.; Strlič, M. Collections Care Scenario Appraisal for Painting Canvases at Museu Nacional d'Art de Catalunya, Barcelona, Spain. Stud. Conserv. 2015, 60, 193-199.

(34) Mecklenburg, M. F. Some Aspects of the Mechanical Behavior of Fabric Supported Paintings; Report to the Smithsonian Institution, 1982; pp 12-15. 
(35) Beamon, G.; Briggs, D. High Resolution XPS of Organic Polymers: The Scienta ESCA300 Database. J. Chem. Educ. 1993, 70 (1), 25.

(36) Pierce, M.; Stuart, J.; Pungor, A.; Dryden, P.; Hlady, V. Adhesion Force Measurements Using an Atomic Force Microscope Upgraded with a Linear Position Sensitive Detector. Langmuir 1994, 10 (9), 3217-3221.

(37) Leite, F. L.; Bueno, C. C.; Da Róz, A. L.; Ziemath, E. C.; Oliveira, O. N. Theoretical Models for Surface Forces and Adhesion and Their Measurement Using Atomic Force Microscopy. Int. J. Mol. Sci. 2012, 13, 12773-12856.

(38) Nechyporchuk, O.; Yu, J.; Nierstrasz, V. A.; Bordes, R. Cellulose Nanofibril-Based Coatings of Woven Cotton Fabrics for Improved Inkjet Printing with a Potential in E-Textile Manufacturing. ACS Sustainable Chem. Eng. 2017, 5 (6), 4793-4801.

(39) Andreasson, B.; Forsstrom, J.; Wägberg, L. Determination of Fibre Pore Structure: Influence of Salt, $\mathrm{pH}$ and Conventional Wet Strength Resins. Cellulose 2005, 12, 253.

(40) Wägberg, L.; Björklund, M. On the Mechanism Behind Wet Strength Development in Papers Containing Wet Strength Resin. Nord. Pulp Pap. Res. J. 1993, 8, 53.

(41) Gardner, D.; Oporto, G.; Mills, R.; Samir, M. Adhesion and Surface Issues in Cellulose and Nanocellulose. J. Adhes. Sci. Technol. 2008, 22, 545.

(42) Soignet, D. M.; Berni, R. J.; Benerito, R. R. ESCA - a Tool for Studying Treated Textiles. J. Appl. Polym. Sci. 1976, 20, 2483-2495.

(43) Johansson, L.-S.; Campbell, J.; Koljonen, K.; Kleen, M.; Buchert, J. On Surface Distributions in Natural Cellulose Fibres. Surf. Interface Anal. 2004, 36, 706-710.

(44) Mitchell, R.; Carr, C. M.; Parfitt, M.; Vickerman, J. C.; Jones, C. Surface Chemical Analysis of Raw Cotton Fibres and Associated Materials. Cellulose 2005, 12, 629-639.

(45) Taylor, R. A. Natural Waxes on Cotton Contribute to Yarn and Fabric Quality. Text. Chem. Col. 1997, 29, 32-35.

(46) Johansson, L.-S.; Tammelin, T.; Campbell, J. M.; Setälä, H.; Österberg, M. Experimental Evidence on Medium Driven Cellulose Surface Adaptation Demonstrated Using Nanofibrillated Cellulose. Soft Matter 2011, 7 (22), 10917.

(47) Seery, M. Paper Conservation. Education in Chemistry, 2013 https://eic.rsc.org/feature/paper-conservation/2020204.article.

(48) Siroka, B.; Noisternig, M.; Griesser, U. J.; Bechtold, T. Characterization of Cellulosic Fibers and Fabrics by Sorption/ Desorption. Carbohydr. Res. 2008, 343, 2194-2199.

(49) Lindstrom, T.; Wagberg, L.; Larsson, T. On the Nature of Joint Strength in Paper- a Review of Dry and Wet Strength Resins used in Paper Manufacturing. In Proceedings of 13th Fundamental Research Symposium, Cambridge, Pira International, Leatherhead, 2005; pp 457-562.

(50) Su, J. K.; Mosse, W.; Sharman, S.; Batchelor, W.; Garnier, G. Paper Strength Development and Recyclability with PolyamidoamineEpichlorohydrin (PAE). BioResources 2012, 7 (1), 913-924. 